\title{
MOTIVACIÓN Y ESTIMACIÓN DEL TIEMPO EN EL USO DE HERRAMIENTAS INTERNET INFORMACIONALES Y DIALÓGICAS
}

\author{
Ramón Cladellas-Pros, Luis Cárcamo-Ulloa y Antoni Castelló-Tarrida
}

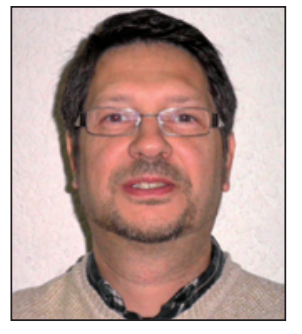

Ramón Cladellas-Pros es profesor lector en el Departamento de Psicología Básica, Evolutiva y de la Educación (Facultad de Psicología) de la Universidad Autónoma de Barcelona (UAB). Doctor y licenciado en psicología. Da clases en las facultades de Psicología y Periodismo y en el programa de Doctorado en percepción, comunicación y tiempo de la UAB. Su línea de investigación se orienta al estudio de los procesos de percepción temporal, tratamiento de la información y comunicación y procesos cognitivos.

Departamento de Psicología Básica, Evolutiva y de la Educación Facultad de Psicología. Edif. B - Campus de la UAB 08193 Bellaterra (Cerdanyola del Vallès). Barcelona, Spain Tel.: +34-935 814 531; fax +34-935813 329 ramon.cladellas@uab.es

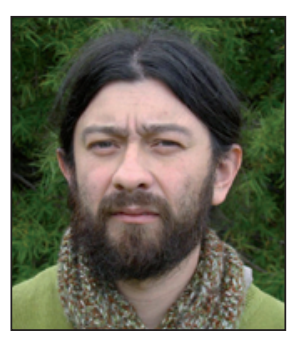

Luis Cárcamo-Ulloa es profesor en el Instituto de Comunicación Social de la Universidad Austral de Chile (UACH). Licenciado en comunicación por la UACH y doctor en percepción comunicación y tiempos por la Univ. Autónoma de Barcelona. Da clases de psicología de la comunicación y comunicación multimedia en el pregrado de periodismo y dirige el programa de Magíster en Comunicación en la $\mathrm{UACH}$. Investiga en procesos informacionales y dialógicos mediados por tecnologías de la información y las comunicaciones.

Facultad de Filosofía y Humanidades. Universidad Austral de Chile. Instituto de Comunicación Social Av. Elena Haverberk, casilla 576 Campus Isla Teja, s/n. Valdivia, Chile Icarcamo@uach.cl

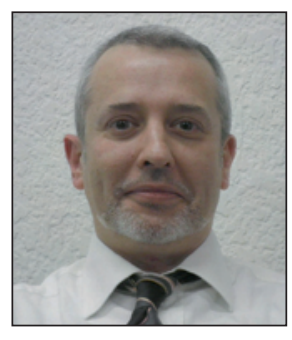

Antoni Castelló-Tarrida es profesor titular en el Departamento de Psicología Básica, Evolutiva y de la Educación (Facultad de Psicología) de la Universidad Autónoma de Barcelona (UAB). Doctor y licenciado en psicología. Su línea de investigación se orienta al estudio de la inteligencia, procesamiento de información y procesos cognitivos.

Departamento de Psicología Básica, Evolutiva y de la Educación Facultad de Psicología. Edif. B - Campus de la UAB 08193 Bellaterra (Cerdanyola del Vallès). Barcelona, Spain Tel.: +34-935 812 101; fax +34-935813 329 toni.castello@uab.es

\section{Resumen}

Se analiza la relación existente entre el grado de interés o motivación y estimación del tiempo (aspectos psicológicos) en la realización de tareas informacionales y dialógicas (aspectos comunicacionales) mediadas por internet en adolescentes chilenos. Se empleó una muestra estratificada de 120 estudiantes de 7 liceos de Chile con una media de edad de 15 años. Todos los participantes debían realizar de forma sucesiva dos tareas por internet: una primera tarea de comunicación vertical (búsqueda de información con el empleo del Google) y una segunda tarea de comunicación horizontal (diálogo por mensajería). Para cada una de las tareas se medía el tiempo percibido y el grado de interés. Los resultados muestran un interés significativamente mayor y un tiempo estimado significativamente menor en la tarea de comunicación horizontal. Desde aquí se pueden proyectar usos informacionales y educativos de internet.

\section{Palabras clave}

Motivación, TIC, Internet, Estimación del tiempo, Tarea de búsqueda, Tarea de mensajería.

Title: Motivation and time estimates in informational and dialogical internet tools usage

\begin{abstract}
This paper analyzes the relationship between motivation and time estimates spent on internet based tasks, and its eventual incidence in informational and dialogical activities. The sample consisted of 120 students from 7 schools ("Ii-
\end{abstract}


ceos") in Chile, whose average age was 15. The participants had to carry out two different tasks on the Internet: first, a vertical communication activity (Google search); second, a horizontal communication activity (messaging). In each task, the perceived elapsed time and the level of interest were evaluated. When comparing the vertical and the horizontal communication activities, the results show a significantly higher interest and significantly lower estimated time in the horizontal communication task (chatting). From these results, a projection of the informational and educational uses of the internet can be made.

\section{Keywords}

Motivation, ICTs, Internet, Time estimates, Search task, Chat task.

Cladellas-Pros, Ramón; Cárcamo-Ulloa, Luis; Castelló-Tarrida, Antoni. “Motivación y estimación del tiempo en el uso de herramientas internet informacionales y dialógicas". El profesional de la información, 2011, enero-febrero, v. 20, n. 1 , pp. 25-31.

DOI: 10.3145/epi.2011.ene.03

\section{Introducción e investigaciones previas}

Desde diferentes posiciones teóricas se ha enfatizado la importancia de atender conjuntamente los aspectos cognitivos y motivacionales implicados en el aprendizaje (Braten; Olaussen, 1998; Pintrich, 2000; Rinaudo; Chiecher; Donolo, 2003).

Lo que no se sabe es cómo interactúan tales aspectos temporales en la realización de tareas por internet, y las consecuencias que de ello se pueden desprender. Cuando se habla de web 2.0, "la auténtica revolución viene de la concepción que se tiene de los usuarios: se ha pasado de hablar de ellos como consumidores de información a tratarlos en términos de carácter participativo en la elaboración y gestión de los contenidos" (Margaix-Arnal, 2007).

La motivación puede definirse como un proceso psicológico que activa el comportamiento y lo dirige a la consecución de ciertos objetivos o metas más o menos difíciles de lograr (Nuttin, 1982). Aunque suele diferenciarse entre motivaciones intrínsecas y extrínsecas (Deci; Ryan, 2000), ambas tienen como función principal el impulso y el mantenimiento del comportamiento.

El tiempo psicológico o subjetivo es la experiencia interna del paso del tiempo, o de cuánto tiempo se cree que ha transcurrido desde la ocurrencia de algún evento. El paso del tiempo psicológico, percibido o subjetivo depende no sólo de factores biológicos, sino también del aprendizaje, habilidad cognitiva, experiencia, ambiente físico y social, personalidad, cultura y otros.

La experiencia subjetiva del paso del tiempo es un aspecto central de la experiencia motivacional. Así, según Csikszentmihalyi, cuando una persona está plenamente involucrada en una tarea que encuentra interesante experimenta una sensación rápida del paso del tiempo (Csikszentmihalyi, 1975; 1990; Csikszentmihalyi; Rathunde, 1993). En el terreno educacional una actividad de aprendizaje divertida, acompañada por una baja estimación del paso del tiempo, tiende a repetirse entre aquellos estudiantes que previamente la han experimentado. De este modo se genera un ciclo en que este estado placentero actúa como elemento motivacional que permite desarrollar hábitos que les ayudan a no estar pendientes del tiempo y les induce a una mayor concentración en aquello que realizan. En la misma línea, Conti (2001) sostiene que los estudiantes motivados tienden a estar menos pendientes del tiempo y con frecuencia experimentan su paso de forma más rápida.

Los modelos que tradicionalmente explican la percepción del tiempo en la realización de tareas comprenden factores como la memoria (Ornstein, 1969), la cantidad de estímulos (Pointer, 1989), la interacción del sujeto en la actividad (Orsini, 1971) y la calidad de tiempos llenos o vacíos que implica la tarea (Gambara; Botella; Gemp, 2002).

Por otra parte, Morales (2005) propone un modelo teórico específico para evaluar la estimación del tiempo en el trabajo con tecnologías, en el que considera el dominio de la tarea, la cantidad de información que ésta implica y la alerta puesta en la misma. El estudio de Mumtaz (2001) con niños de escuela primaria, explica que la actividad más popular y entretenida que ejecutan los chicos en los ordenadores desde sus hogares está relacionada con los videojuegos, mientras que en los ordenadores de la escuela es el procesamiento de textos, tarea que consideran aburrida.

Según Pintrich y otros (1991) y Wolters y Pintrich (1998), una alta valoración de las tareas a realizar podría provocar que el estudiante se involucre más en el propio aprendizaje y utilice con más frecuencia estrategias cognitivas. En este mismo sentido, McRobbie y Tobin (1997) argumentan que cuando las tareas académicas son percibidas como interesantes, importantes y útiles, los estudiantes pueden estar más dispuestos a aprender con comprensión.

Los avances de las tecnologías de la información y comunicación (TICS) han producido cambios sustanciales en la sociedad. En el caso de Chile se ha acentuado con la entrada a principios de los años 90 del programa Enlaces. Desde entonces los ordenadores e internet ocupan un lugar importante en la escuela y para muchos niños es el primer encuentro con la red de redes. Además el impacto de las TICs en los diferentes niveles del sistema educativo ha sido objeto de numerosos estudios evaluativos en los últimos años (Cuban, 2001; Area, 2005). La integración de las TICs en espacios escolares se observa a partir de: cuantificación de su uso en los diferentes niveles de enseñanza, integración e influencia de las mismas en el propio proceso educativo, metodologías empleadas, eficacia de su implantación en la motivación y rendimiento del alumno, etc. El proceso de aprendizaje del alumnado se ha visto modificado: los aspectos que los es- 
colares buscaban antes en enciclopedias de papel, hoy los encuentran en Google, alternativa que les exige la habilidad extra de discriminar. Por otro lado la mensajería o chat no es más rápida que el teléfono, pero sí tiene una condición de permanencia distinta, pues prácticamente los usuarios permanecen disponibles o semi-disponibles mientras están conectados a la Red.

Internet se transforma en un objeto-fin que ofrece varias alternativas a la vez. Es un gran espacio para la exploración e intercambio de conocimientos, además de ser un lugar para conocer y dialogar con otros sujetos. Desde la comunicación se pueden sintetizar en dos grandes grupos las acciones que se llevan a cabo en internet: las tareas de naturaleza exploratoria o de acceso a la información (descargas de archivos y búsquedas de información), y las dialógicas (mensajería instantánea, foros y correo electrónico, entre otras). La evolución de los usos de las tecnologías en Chile (Injuv, 2002; Godoy, 2006) ha pasado de un mayor predominio de tareas orientadas a la búsqueda de información, a un uso cada vez más extendido de tareas que suponen una interacción entre personas.

Internet se transforma en un objeto-fin que ofrece varias alternativas a la vez

En síntesis, tanto para explorar, accediendo verticalmente a la información (Shannon; Weaver, 1949), como para interactuar, estableciendo comunicación horizontal o dialógica (Kaplún, 1998; Rogers, 1986), el ser humano busca en las tecnologías de la información y las comunicaciones el puente que permita satisfacer sus intereses y/o necesidades. Las tecnologías de la información, como característica principal de los tiempos actuales, ofrecen una gran variedad de servicios (páginas web, correo electrónico, chats, programas para descargar música, vídeos y software, etc.) que son fundamentales en el proceso de enseñanza-aprendizaje (Palomares-Casado et al., 2007). Con estas herramientas se pueden experimentar nuevas y más ricas relaciones cognitivas, elaborar distintos esquemas de acción, estudiar modelos de aprendizaje innovadores y recodificar continuamente nuestra teoría del conocimiento (Gramigna; González, 2009). Todo ello con el propósito de crear entre el alumnado la necesidad de acceder a la información y comunicarse a la máxima velocidad y desde cualquier sitio.

El empleo de las TICs en la escuela genera una necesidad de estimulación y de contacto directo con las cosas. Desde la infancia se vive la curiosidad por los objetos desconocidos o menos conocidos. La novedad se asienta también como fuente de estimulación. Esta situación explicaría los impulsos que invocan a reconocer y explorar el medio propio de una forma placentera, y a percibir el paso del tiempo de forma más lenta.

Todo ello nos induce a preguntamos hasta qué punto las demandas cognitivas implicadas en diferentes tareas (naturaleza exploratoria y dialógica) inciden sobre el grado de interés mostrado por la tarea y el tiempo estimado durante la realización de la misma. Si tenemos en cuenta las dimensio- nes cognitivas propuestas en el modelo de Morales (2005), es de esperar que una tarea de naturaleza horizontal-dialógica, en que el usuario debe estar en permanente estado de diálogo y, por tanto, con una mayor demanda de recursos de atención y concentración, requiera un mayor grado de interés y un menor tiempo estimado.

\section{Diseño del experimento}

Como marco de muestreo se contó con un listado de centros públicos de secundaria de las diferentes regiones de Chile comprendidas entre Santiago y Puerto Montt. Se trataba de evaluar a la población socio-económicamente más vulnerable y que accede a internet básicamente en la escuela. La estructura de la población de muestreo está conformada por estratos socio-económicos medios-bajos y bajos, según las clasificaciones del Simce (Sistema de medición de la calidad de la educación chilena). Así las unidades de estudio dentro de cada estrato presentan características similares.

Una vez estratificada la población, se calculó el tamaño de la muestra requerida de liceos resultando un total de $7 \mathrm{li}$ ceos ubicados en diferentes regiones chilenas. En una fase posterior se decidió obtener una muestra aleatoria de estudiantes de cada liceo. Las restricciones del número de alumnos por centro se debieron a la limitación del número de equipos informáticos. La muestra final estaba formada 120 estudiantes, de los cuales $62(51,7 \%)$ son mujeres y 58 $(48,3 \%)$ hombres. La edad media era de 15,3 años.

La muestra final estaba formada por 120 estudiantes, de los cuales $62(51,7 \%)$ son mujeres y $58(48,3 \%)$ hombres. La edad media era de 15,3 años

\subsection{Instrumentos de recogida de datos \\ Cuestionario de búsqueda de información}

Se compone de dos partes. La primera contiene las instrucciones para buscar información relevante: lugar y fecha de nacimiento, principales obras y lugar y fecha de fallecimiento de cada uno de los seis personajes propuestos (Pablo de Rocka, George Harrison, Carl Lewis, Martin Luther King, Francis Crick y John Nash). Se escogieron seis personajes de diferentes ámbitos, para diversificar el posible conocimiento de los participantes en un área determinada. En la segunda parte el sujeto tenía que dejar constancia de lo encontrado y contestar una serie de preguntas, entre las que figuraba el tiempo que pensaba que había transcurrido durante la realización de la tarea y el grado de interés que le había suscitado la misma.

\section{Cuestionario de diálogo mediado por mensajería instantá- nea}

Se compone también de dos partes: una primera con las instrucciones de la tarea a realizar; y una segunda con una hoja de respuestas, entre las que se encontraba el tiempo que pensaban que había transcurrido desde el inicio de la tarea y el grado de interés que los motivaba. 


\subsection{Materiales}

Se emplearon los ordenadores que se encontraban en el laboratorio de la red Enlaces de los colegios, para que las condiciones experimentales fueran conocidas e iguales para todos los participantes. En todos los casos la conectividad a internet resultaba estable, equivalente a banda ancha de 512 Kbps o similar.

Para efectos de las actividades experimentales, el sistema operativo en todos los casos era Windows XP y los estudiantes realizaron la prueba de "búsqueda de información" en Google con un navegador Explorer 6.2 y la prueba de "diálogo mediado por mensajería instantánea" con Messenger 6.2 .

Se intentó que las condiciones experimentales fueran conocidas e iguales para todos los participantes

\subsection{Variables}

\section{Dependientes:}

- Grado de interés: nivel de motivación manifestado en la realización de las diferentes tareas. Se evaluaba de acuerdo con las respuestas proporcionadas en la siguiente proposición tipo Lickert "Califica de 1 a 5 el interés mostrado por la experiencia". La mínima puntuación de 1 se correspondía a "nada interesante" y la máxima puntuación de 5 a "muy interesante".

- Tiempo estimado: número de segundos que el participante indicó haber percibido desde el inicio hasta el final de la tarea. El tiempo real destinado a la realización de la tarea era de $8,2 \mathrm{~min}$ (490 segundos).

Independiente:

- Tipo de tarea variable con dos categorías: tarea vertical-informacional (búsqueda de información) y tarea horizontaldialógica (diálogo mediado por mensajería instantánea). Se trataba de realizar una búsqueda de información con Google; y mantener una conversación con otra persona vía chat (estudiantes universitarios previamente entrenados para sostener la conversación durante la experimentación).

Además se controlaron las variables de edad y de género en los participantes, para configurar grupos de escolares de características homogéneas.

\subsection{Procedimiento}

En una primera fase pre-experimental o fase piloto se visitó un total de 16 liceos con el objetivo de analizar, revisar y superar posibles incidencias que se pudieran producir, especialmente las referidas a control de variables o condiciones de los laboratorios informáticos.
De todas las variables estudiadas se puso especial énfasis en dos: tiempo total disponible para realizar la tarea y frecuencia semanal de acceso a la misma. Así se pudo concluir que un tiempo de 8,2 minutos garantizaba un tiempo completo de actividad; y se verificó que todos los sujetos, independientemente de la zona en que se encontraban los liceos, tuvieran un acceso frecuente a las tecnologías en sus centros educativos. Las frecuencias medias oscilaban de 3,3 días a la semana en la prueba de búsqueda de información, a 3,6 en la de mensajería.

En la fase experimental participó un total de 120 sujetos que habían pasado previamente por la fase pre-experimental. Se descartaron los que en promedio usaban alguna de las herramientas (buscadores de información o mensajería instantánea) menos de dos veces por semana.

El procedimiento para los participantes era siempre el mismo. Tenían que realizar dos actividades sucesivas: una de búsqueda de información y otra de diálogo mediado por mensajería instantánea. Cada actividad se realizaba durante un tiempo de 8 minutos y 10 segundos. Las sesiones se realizaban en grupos de seis personas en un laboratorio con ordenadores. La búsqueda de información se realizaba en Google (figura 1). Como ya se ha comentado, en esta tarea los participantes tenían que buscar algunos datos biográficos de los 6 personajes seleccionados; mientras que para la tarea de diálogo mediado por mensajería instantánea se utilizó el software MSN (figura 2) y los sujetos interactuaban con un personaje cuya identidad desconocían. La persona que actuaba como monitor experto era un alumno que había sido entrenado previamente. Éste debía seguir estrictamente un protocolo con una serie de instrucciones, con la finalidad de asegurar la dinámica del experimento y el desarrollo de un tiempo completo de esta actividad.

Se realizaron dos actividades sucesivas: búsqueda de información y diálogo mediado por mensajería instantánea

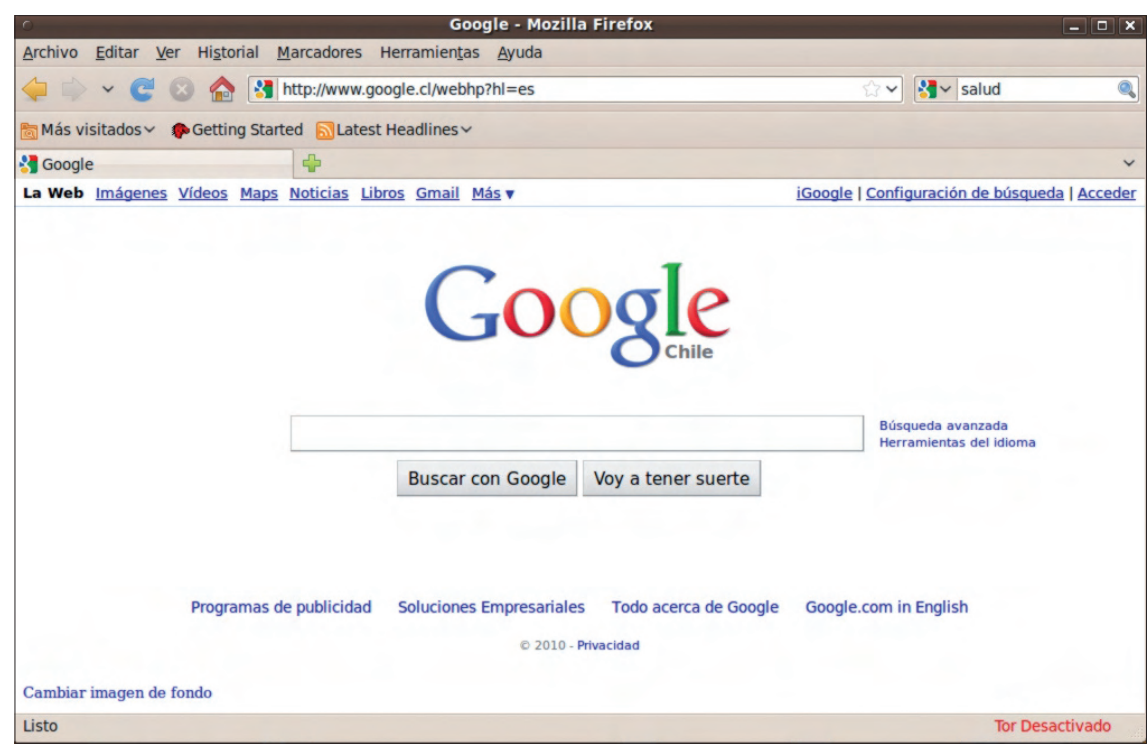

Figura 1: Software empleado para la búsqueda de información 


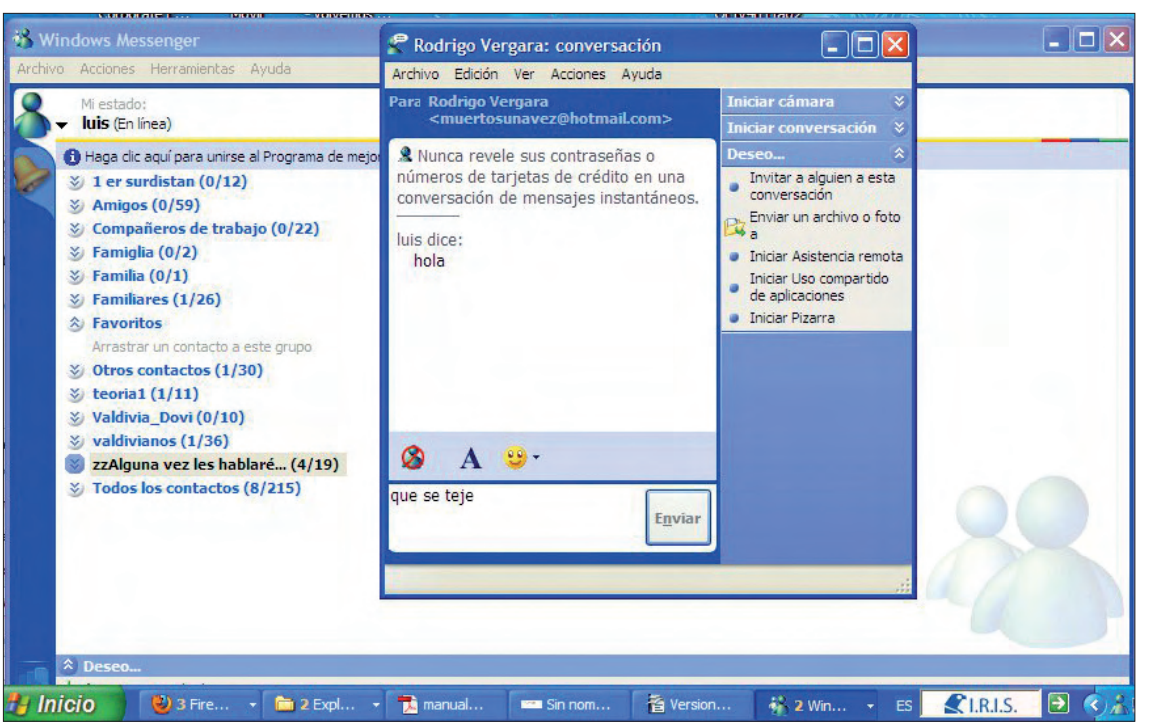

Para comprobar la hipótesis referente al grado de interés mostrado en la realización de las tareas se realizó una prueba $t$ de Student para muestras relacionadas. La variable dependiente es el grado de interés y la variable independiente corresponde a la naturaleza de las características de las tareas (búsqueda y diálogo).

Como se observa en la tabla 2, la media del grado de interés para la tarea de "búsqueda de información" es significativamente inferior $(p=0,000)$ que el manifestado en la tarea de "diálogo mediado por mensajería instantánea".

Figura 2: Software empleado para la tarea de mensajería

Entre grupo y grupo se invertía el orden de ejecución de las tareas, con el propósito de evitar que ello pudiera influir en los resultados.

La recogida de datos se realizó mediante los dos cuestionarios antes mencionados. Los investigadores se desplazaron a los diferentes liceos. Antes de comenzar cada sesión experimental se explicaban las instrucciones de las tareas a realizar y se garantizaba la confidencialidad de los datos aportados. La participación en el estudio fue totalmente voluntaria, siguiendo los protocolos de ética dictados por el Fondecyt (Fondo Nacional de Desarrollo Científico y Tecnológico).

\section{Resultados}

Para comprobar si la naturaleza de la tarea (búsqueda o diálogo) llevada a cabo con ordenadores incidía sobre la estimación del tiempo que hacen los sujetos -siendo subestimado el tiempo para la tarea de diálogo mediado por mensajería instantánea frente a la estimación temporal hecha en la tarea búsqueda de información-, se realizó una prueba $t$ de Student ${ }^{1}$ para muestras relacionadas. La variable dependiente es la estimación de tiempo y la variable independiente se corresponde con la naturaleza de las características de las tareas (búsqueda y diálogo).

En el conjunto de los resultados se constata cómo el tiempo estimado por los participantes en cada una de las variables estudiadas sobrepasa el tiempo real de 490 segundos. Un análisis entre los tipos de tarea manifiesta diferencias significativas $(p=0,000)$. Tal y como se puede observar en la tabla 1 , el tiempo estimado en la tarea de búsqueda de información $(688,72 \mathrm{seg})$ es superior al de la tarea de mensajería (588,28 seg).

\begin{tabular}{|c|c|c|c|c|}
\hline \multicolumn{1}{|c|}{ Tarea } & $\begin{array}{c}\text { Media de } \\
\text { tiempo }\end{array}$ & $\mathbf{N}$ & $\begin{array}{c}\text { Desviación } \\
\text { típica }\end{array}$ & $\mathbf{t}$ \\
\cline { 1 - 4 } Búsqueda de información & 688,72 & 120 & 308,07 & \multirow{2}{*}{4,062} \\
\hline Diálogo por mensajería & 586,28 & 120 & 275,65 & \\
\hline
\end{tabular}

Tabla 1. Medias del tiempo estimado entre tareas

\begin{tabular}{|c|c|c|c|c|}
\hline Tarea & $\begin{array}{c}\text { Media de } \\
\text { interés }\end{array}$ & $\mathbf{N}$ & $\begin{array}{c}\text { Desviación } \\
\text { típica }\end{array}$ & $\mathbf{t}$ \\
\hline Búsqueda de información & 3,69 & 120 & 0,977 & \multirow{2}{*}{$-6,116$} \\
\hline Diálogo por mensajería & 4,34 & 120 & 0,912 & \\
\hline
\end{tabular}

Tabla 2. Medias del grado de interés entre tareas

Para comprobar la relación entre ambas variables (tiempo estimado y grado de interés) se realizó un análisis de correlaciones bivariadas. La correlación negativa de $0,18(p<0,01)$ establece indicios de direcciones opuestas entre ambas variables; así, cuanto mayor es el grado de interés mostrado en la realización de una tarea menor es el tiempo estimado, tal y como se puede observar en la tabla 3.

\begin{tabular}{|l|c|c|}
\hline \multicolumn{1}{|c|}{ Tarea } & $\begin{array}{c}\text { Tiempo } \\
\text { estimado }\end{array}$ & Interés \\
\hline Búsqueda de información & 688,72 & 3,69 \\
\hline Diálogo por mensajería & 586,28 & 4,34 \\
\hline
\end{tabular}

Tabla 3. Resumen de medias de las variables

El tiempo estimado en la tarea de búsqueda de información $(688,72 \mathrm{seg})$ es superior al de la tarea de mensajería (588,28 seg)

\section{Discusión y conclusiones}

En términos generales y analizando descriptivamente los datos, los procesos de estimación del tiempo tienden a ser sobreestimados. El tiempo no pasa volando, al menos en los ocho minutos y diez segundos que comprenden las actividades experimentales llevadas a cabo.

De los resultados se desprende que las tareas más dialógicas u horizontales son consideradas las más interesantes y las que provocan una mayor subestimación del paso del 
tiempo. Asimismo, se comprueban efectos contrarios entre ambas variables (interés y estimación del tiempo). De acuerdo con los resultados encontrados por Csikszentmihalyi y Rathunde (1993), las tareas consideradas más interesantes provocan una sensación del paso del tiempo más lento.

Como plantean Reparaz, Sobrino y Mir (2001), las nuevas tecnologías están revolucionando el mundo de las comunicaciones, el receptor es quien selecciona la información que desea recibir y la forma como quiere obtenerla. Por ello sería ideal que en esta nueva etapa pudieran encontrarse usos más aplicados y variados de las tecnologías de la información y las comunicaciones, y que para la búsqueda de nuevos procesos se tuviera en cuenta la mayor motivación generada por los procesos de diálogo mediado por mensajería instantánea. En un sentido convergente López-Hernández y Penadés-De-la-Cruz (2007) sugieren por ejemplo herramientas aplicadas a la bibliotecología que permitan "acercarnos a una de las características fundamentales de la web 2.0 como es la participación".

Las implicaciones informacionales y educacionales de este estudio pasan por considerar aquellas tareas que puedan ser más interesantes o motivantes para los alumnos. Durante su realización ellos perciben el paso del tiempo de forma rápida, y por ende, experimentan una menor sensación de cansancio. Esta disminución de sensación de esfuerzo posibilita que una misma tarea (dependiendo del grado de interés o motivación) se pueda realizar durante un período de tiempo más prolongado, y por tanto el alumno pueda tener más tiempo de contacto con los contenidos del aprendizaje, repercutiendo ello en un aprendizaje académico más efectivo.

De los resultados se desprende que las tareas dialógicas u horizontales son consideradas más interesantes

A la luz de los resultados obtenidos en este experimento se sugiere la necesidad de implementar tareas de interacción entre los estudiantes. Para ello se requiere la implantación de recursos educativos como chats, forums o espacios virtuales docentes, en los que los jóvenes puedan aprender e interactuar colectivamente. Si se consigue que estos espacios docentes sean efectivos, el alumno será capaz de destinar un considerable número de horas a una misma tarea, sin notar los efectos del cansancio, y de una forma divertida.

Los nuevos desarrollos tecnológicos hacen posible el estudio de nuevos procesos interactivos que se instalan y desinstalan no sólo de los computadores sino también de los círculos sociales estableciendo formas de comportamiento colectivo y social (Flores-Vivar, 2009). El cambio y la innovación son aceptados por los usuarios de la Red como una situación permanente. Los fotologs son un ejemplo claro de esas interacciones comunicacionales. Hace cinco años casi no existían, hoy se trata de una actividad recurrente entre adolescentes y universitarios. Se observa otro fenómeno a partir de la posibilidad de compartir vídeos en servidores de streaming como YouTube o GoogleVideo (Cárcamo-Ulloa,
2008) que si bien no se trata de redes sociales atraen una gran cantidad de adeptos entre la juventud.

\section{Limitaciones del trabajo}

Este tipo de estudios no está exento de limitaciones, pues Chile es un país con una gran variedad geográfica, climatológica y cultural. Estos factores convierten al territorio en una mina de pluralidad para la aplicación de estudios comparativos, y los prolongados desplazamientos y la escasa accesibilidad a algunos territorios encarecen y prolongan las condiciones para un macroestudio. Por todo lo anterior, las comparaciones presentadas en esta investigación tienen gran valor para los grupos investigados pero no necesariamente se pueden generalizar a otros grupos.

\section{Notas}

1. La distribución $t$ de Student fue descrita en 1908 por William S. Gosset. Gosset trabajaba en la fábrica de cerveza Guinness, que prohibía a sus empleados la publicación de artículos científicos debido a una difusión previa de secretos industriales. De ahí que Gosset publicase sus resultados bajo el seudónimo Student.

2. Este artículo forma parte de los resultados del Proyecto Fondecyt 11080193 (Fondo Nacional de Desarrollo Científico y Tecnológico de Chile).

\section{Bibliografía citada}

Area, Manuel. "Las tecnologías de la información y comunicación en el sistema escolar. Una revisión de las líneas de investigación". Revista electrónica de evaluación educativa, 2005 , v. 11, n. 1, pp. 3-25.

http://www.uv.es/relieve/v11n1/RELIEVEv11n1_1.htm

Braten, Ivar; Olaussen, Bodil. "The relationship between motivational beliefs and learning strategy use among Norwegian college students". Contemporary educational psychology, 1998, v. 23, n. 2, pp. 182-194.

Cárcamo-Ulloa, Luis. Estimación de tiempo en estudiantes secundarios chilenos frente a tareas de búsqueda de información y comunicación desarrolladas con apoyo de internet. Tesis doctoral, Universidad Autónoma de Barcelona, 2008.

Conti, Regina. "Time flies: investigating the connection between intrinsic motivation and the experience of time". Journal of personality, 2001, v. 69, n. 1, pp. 1-26.

http://edtech.cebs.wku.edu/ rmiller/wpi.pdf

Csikszentmihalyi, Mihály. Beyond boredom and anxiety: experiencing flow in work and play. San Francisco, CA: JosseyBass, 1975, ISBN 0787951404.

Csikszentmihalyi, Mihály. Flow: the psychology of optimal experience. New York: Harper \& Row, 1990, ISBN 0060920432.

Csikszentmihalyi, Mihály; Rathunde, Kevin. "The measurement of flow in everyday life: toward a theory of emergent motivation". En: Jacobs, J. E. (Editor). Nebraska symposium on motivation. Lincoln: University of Nebraska Press, 1992, v. 40 , pp. 57-97. 
Cuban, Larry. Oversold and underused: computers in the classroom. London: Harvard University, 2001, ISBN 067400602X.

Deci, Edward L.; Ryan, Richard M. "The what and why of goal pursuits: human needs and the self-determination of behavior". Psychological inquiry, 2000, v. 11, n. 4, pp. 227268.

http://www.updatenet.net/images/7/75/Selbstbestimmung_ Deci_Ryan_2000.pdf

Flores-Vivar, Jesús-Miguel. "Nuevos modelos de comunicación, perfiles y tendencias en las redes sociales". Comunicar, 2009, v. 33, pp. 73-81.

http://redalyc.uaemex.mx/pdf/158/15812486009.pdf

Gambara, Hilda; Botella, Juan; Gempp, René. "Tiempo vacío y tiempo lleno. Un meta-análisis sobre los cambios en la percepción del tiempo en la edad". Revista estudios de psicología, 2002, v. 23, n. 1, pp. 87-100.

http://www.sigmas.cl/papers/MetanalisisTiempo.pdf

Godoy, Sergio. Monitoreando el futuro digital: resultados encuesta WIP-Chile 2006. World International Project, 2006.

http://comunicaciones.uc.cl/prontus fcom/site/artic/2008 0418/asocfile/20080418230431/wip_chile_2006_informe_ final_en_pdf_ok.pdf

Gramigna, Anita; González-Faraco, Juan-Carlos. "Videojugando se aprende: renovar la teoría del conocimiento y la educación". Comunicar, 2009, v. 17, n. 33, pp. 157-164.

http://redalyc.uaemex.mx/pdf/158/15812486019.pdf

Injuv. Transformaciones culturales e identidad juvenil en Chile. Temas de desarrollo humano sustentable, n. 9. Chile: PNUD, 2002.

http://www.desarrollohumano.cl/otraspub/pub09/pnud_ injuv.pdf

Kaplún, Mario. Una pedagogía de la comunicación. Madrid: Ediciones de la Torre, 1998, ISBN 847960185X.

López-Hernández, Francisco; Penadés-De-la-Cruz, Honorio. "365 días de libros: blog para la promoción de la lectura". El profesional de la información, 2007, marzo-abril, v. 16, n. 2, pp. 131-133.

Margaix-Arnal, Dídac. "Conceptos de web 2.0 y biblioteca 2.0: origen, definiciones y retos para las bibliotecas actuales". El profesional de la información, 2007, marzo-abril, v. 16, n. 2, pp. 95-106.

http://www.oei.es/tic/kx5j65q110j51203.pdf

McRobbie, Campbell; Tobin, Kenneth. "A social constructivist perspective on learning environments". Intl journal of science education, 1997, v. 19, n. 2, pp. 193-208.

Morales-Velázquez, Cesareo. "Tiempo psicológico en los estudiantes y carga de información". Ingenierías, 2005, v. 8, n. 27, pp. 16-23.

http://www.ingenierias.uanl.mx/27/27_tiempo_psico.pdf

Mumtaz, Shazia. “Children's enjoyment and perception of computer use in the home and the school". Computers \& education, 2001, v. 36, n. 4, pp. 347-362.

Nuttin, Joseph. Teoría de la motivación humana. Barcelona: Paidós, 1982, ISBN 8475091563.

Ornstein, Robert. On the experience of time. New York, USA: Penguin Books, 1969, ISBN 081333442X

Orsini, Francine. "Contribución al estudio genético de la estimación del tiempo en función de la variación de las situaciones". En: Piaget, Jean; Grize, Jean-Blaise. La epistemología del tiempo. Buenos Aires: Editorial El Ateneo, 1971, pp. 162-190.

Palomares-Casado, Teodoro; Fernández-Aguirre, Karmele; Madroño-Herrán, Juan; González-Velasco, Javier; SáezCrespo, Francisco; Chica-Páez, Yolanda; Torres-Barañano, Amelia; Chomón-Villanueva, María-Jesús; Bilbao-Zulaica, Pedro. "Las tecnologías de la información y comunicación en la enseñanza universitaria: influencia sobre la motivación, el autoaprendizaje y la participación activa del alumno". Revista de psicodidáctica, 2007, v. 12, n. 1, pp. 51-78. http://redalyc.uaemex.mx/pdf/175/17512104.pdf

Pintrich, Paul; Smith, David; García, Teresa; McKeachie, Wilbert J. A manual for the use of the motivated strategies for learning questionnaire (MSLQ). National Center for Research to Improve Postsecondary Teaching and Learning. University of Michigan, 1991, Grant number OERI-86-0010. http://www.eric.ed.gov/PDFS/ED338122.pdf

Pintrich, Paul. "The role of goal orientation in self-regulated learning". En: Boekaerts, Monique; Pintrich, Paul; Zeidner, Moshe (editores). Handbook of self-regulation, San Diego: Academic Press, 2000, pp. 452-502. ISBN 0121098907.

Pointer, Douglas. "Judging the duration of time intervals: a process of remembering segments of experience". En: Levin, Iris; Zakay, Dan (eds.). Time and human cognition. Netherlands: Advanced in psychology, 1989, v. 59, pp. 304-331. ISBN 044487379I.

Repáraz, Charo; Sobrino, Ángel; Mir, José. “Integración curricular de las nuevas tecnologías”. Barcelona, Ariel, 2001, 165 pp. ISBN 8434428717.

Rinaudo, María-Cristina; Chiecher, Analía; Donolo, Danilo. "Motivación y uso de estrategias en estudiantes universitarios. Su evaluación a partir del Motivated Strategies Learning Questionnaire". Anales de psicología, 2003, v. 19, n. 1, pp. 107-119.

http://www.um.es/analesps/v19/v19_1/11-19_1.pdf

Rogers, Everett. Communication technology: the new media in society. New York: Free Press. 1986. ISBN 002927110X.

Shannon, Claude; Weaver, Warren. The mathematical theory of communication. USA: University of Illinois Press, 1949.

Wolters, Christopher; Pintrich, Paul. "Contextual differences in student motivation and self-regulated learning in mathematics, English and social studies classrooms". Instructional science, 1998 , v. 26, n. 1-2, pp. 27-47. 